Original article

\title{
Influencing variables for fetal growth in malnourished mothers: A nested case-control study
}

\author{
Neha $^{\mathrm{a}}$, Suchetha S. Rao ${ }^{\mathrm{a}}$, B. Shantharam Baliga ${ }^{\mathrm{a}}$, Prasanna Mithra ${ }^{\mathrm{b}}$, Poornima Manjrekar ${ }^{\mathrm{c}}$, \\ Nutan Kamath ${ }^{\mathrm{a}, *}$ \\ ${ }^{a}$ Department of Paediatrics, Kasturba Medical College, Mangalore, Manipal Academy of Higher Education, Manipal, Karnataka, India \\ ${ }^{\mathrm{b}}$ Department of Community Medicine,Kasturba Medical College, Mangalore, Manipal Academy of Higher Education, Manipal, Karnataka, India \\ ${ }^{\mathrm{c}}$ Department of Biochemistry, Kasturba Medical College, Mangalore, Manipal Academy of Higher Education, Manipal, Karnataka, India
}

\section{A R T I C L E I N F O}

\section{Keywords:}

Anemia

Body mass index

Ferritin

Low birth weight

Maternal malnutrition

\begin{abstract}
A B S T R A C T
Objective: Maternal malnutrition, inadequate weight gain in pregnancy, and maternal anemia are the modifiable variables commonly implicated for intrauterine growth retardation in the community. We aimed to evaluate the frequency of growth retardation and iron deficiency among babies born to malnourished mothers and its association with maternal anthropometry, hemoglobin, and ferritin.

Methods: A nested case-control study was carried out as analysis of subset data with subjects enrolled for a randomized controlled trial(CTRI Trial no.2018/04/013096)in government facilities of Kasturba Medical College, Mangalore, affiliated to Manipal Academy of Higher Education from May to August 2018. Eighty-eight newborns born to malnourished primigravidae by vaginal delivery were included. Neonatal anthropometry and cord blood ferritin along with maternal hemoglobin and ferritin was measured. The maternal pre-pregnancy body mass index (BMI) and weight gain in pregnancy were calculated using documented pre-pregnancy weight, height, and present weight.

Results: The average birth weight was $2.6 \mathrm{~kg}$. (95\% CI, SD = 0.5). Fifty-eight percent of babies were appropriate for gestational age(AGA). The number of AGA babies born to mothers who had weight gain $7 \mathrm{~kg}$, and above was significantly higher.[Correlation:0.41,p $=0.0001$ ]. Nine babies had depleted iron stores, three babies being small for gestational age(SGA). (RR:0.77,95\% CI $=0.29-2.05, \mathrm{p}=0.72$ ).

Thirty-four percent mothers were anemic who had higher chance of having SGA babies. $[\mathrm{RR}=1.47,95 \% \mathrm{CI}$ $0.91-2.38, \mathrm{p}=0.12]$. Thirteen mothers had low ferritin levels and 5 of them had SGA babies[RR $=1.51,95 \%$ $\mathrm{CI}=0.68-3.4, \mathrm{p}=0.33]$. Fifty mothers had pre-pregnancy BMI $<18.5$, out of which 19 had SGA babies. [RR:0.80,95\% CI $=0.49-1.31, \mathrm{p}=0.38]$.

Conclusion: Adequate weight gain in pregnancy can help achieve desired fetal growth in malnourished and anemic mothers.
\end{abstract}

\section{Introduction}

Intrauterine growth restriction, along with malnutrition attributes to $22 \%$ of childhood mortality up to five years of age. ${ }^{1}$ The babies who are small for gestational age(SGA) have expanded the risk of mortality and morbidity, including the inability to grow, delay in neurodevelopment and chronic diseases in adulthood. ${ }^{2}$ It is vitally important to identify the modifiable factors identified with fetal growth retardation.

The mother's nutritional status in the conception period has since long thought to be a key determinant for fetal growth and development. Present literature links maternal malnutrition and inadequate weight gain in pregnancy to intrauterine growth retardation and potential risk of disease in adulthood. ${ }^{2}$

Another stated cause, for intrauterine growth retardation, is anemia. Anemia in pregnancy is categorized based on red cell morphology, genetic basis, and by underlying pathology, which includes decreased production, increased loss, and increased red blood cell destruction. Decreased production is associated with a lack of micronutrients, including iron, vitamin B12, and folic acid. Causes specific to Indian subcontinent are food items with insufficient micronutrients due to plant source, fewer amounts of iron enhancers with more amount of iron inhibitors in the diet including tea, coffee, calcium-rich diet, teenage pregnancy, parasite infestation, poor sanitation, and contaminated drinking water. ${ }^{3}$

\footnotetext{
* Corresponding author.

E-mail address: nutan.kamath@manipal.edu (N. Kamath).
} 
The Government of India has taken anemia very seriously with Health and Family Welfare division working zealously through various schemes to address the malnutrition and anemia. The recent National Iron Plus Initiative launched in 2013 integrates the life cycle of a female starting at as early as six months of life till the end of the reproductive period with oral iron and folic acid(IFA) supplements, irrespective to the nutritional and blood parameters through the aid of various government health care centers. The Accredited social health worker (ASHA) has even been sensitized to check the compliance of the IFA supplementation by home visits. The aim of the National Nutrition Mission to reduce anemia by one third in women in the reproductive group by $2022 .{ }^{3}$

The present figure of $50 \%$ of anemia in pregnant women as per National Family Health Survey 4(2015-2016) has been attributed to various causes including inadequate coverage of IFA supplementation with only $12 \%$ women who have taken the pills for more than 90 days, teenage and multiple pregnancies. ${ }^{4}$ The effect of the supplementation on the hematological profile, excluding these parameters has not been studied so far.

The study was aimed to describe the frequency of growth retardation and iron deficiency among babies born to malnourished mothers and its association with maternal anthropometry, hemoglobin, and ferritin in the Dakshina Karnataka region.

\section{Methodology}

The nested case-control study was carried out as analysis of subset data with subjects enrolled for an ongoing facility based single blinded open randomized control trial.(CTRI Trial no.2018/04/013096). Eighty-eight newborns were included, which were born to malnourished primigravidae admitted for vaginal delivery. Based on the neonatal anthropometry, the babies were classified as small for gestational age(SGA) and appropriate for gestational age(AGA).The SGA babies were considered as cases and AGA babies as controls. It was carried in the government facilities of Kasturba medical college, Mangalore affiliated to Manipal Academy of Higher Education, Manipal. (Lady Goschen hospital) from May to August 2018. Neonates with gross congenital anomalies, NICU stay, and those born to mothers with life-threatening conditions (Pre-eclampsia and eclampsia, uncontrolled blood sugar with HbA1C $>6.5 \%$, uncompensated cardiovascular diseases with NYHA Grade 3 and above), malaria positive were excluded from the study.

The mothers were booked cases and had received Albendazole (400 mg) in the second trimester as per National Iron Plus Initiative Program. Permission for collecting the data was obtained from the medical superintendents of Lady Goshen Hospital. Consent was taken from the mothers after explaining the study in their understandable language using a patient information sheet.

The study group was selected upon satisfying both inclusion and exclusion criteria. Two millilitres cord blood was drawn and transferred to a heparinized vacutainer tube. Four millilitres blood was withdrawn from all the mothers simultaneously and divided equally into heparinized and EDTA vacutainer tubes. The complete hemogram was performed on the Coulter DxH 800 analyzer. The UniCel DxH 800 is a quantitative, automated hematology analyzer which provides counts, reticulocytes, nucleated red cells, and leukocyte 5 part differential counts on whole blood. The serum obtained from the heparinized sample of the mother and the baby after centrifuging was used to measure ferritin with serum ferritin quantitative estimation kit, a commercially available solid phase ELISA kit from DRG Diagnostics, read using a microplate reader, that is, ELX 800 at a wavelength of $450 \mathrm{~nm}$.

The neonate's weight, length, and head circumference were checked at $24 \mathrm{~h}$ of birth.The mother's height and pre-pregnancy weight were collected from the medical records and used for calculating the prepregnancy body mass index(BMI). The present weight was recorded before delivery. The weight gain in pregnancy was calculated by noting the difference between the pre-pregnancy weight and the weight at the time of admission to the hospital before delivery. A questionnaire was administered, which provided information regarding iron intake during pregnancy. Compliance towards IFA tablets by checked by recall method and was defined by the consumption of $100 \mathrm{mg}$ ferrous sulphate twice a day which provided $65 \mathrm{mg}$ of elemental iron and $5 \mathrm{mg}$ folic acid tablet once a day for more than 90 days.

\subsection{Definitions}

- The weight at birth was classified as per Brenelli and Martins curve into SGA and AGA babies. For the head circumference and length, WHO curves were used as a reference.

- A mother with first documented pregnancy weight of less than $45 \mathrm{~kg}$ was termed as malnourished. ${ }^{5}$ BMI was also calculated, and any value less than 18.5 was a marker of the mother being underweight. However, BMI was not used in the case definition. A weight gain in pregnancy less than $7 \mathrm{~kg}$ was termed inadequate, which is the average weight gain of a term pregnancy in India. ${ }^{6,7}$ Definition of anemia in the third trimester included a hemoglobin concentration of less than $11 \mathrm{~g} / \mathrm{dL}^{8}{ }^{8}$

- Iron deficiency in pregnancy was defined as serum ferritin was below $30 \mu \mathrm{g} / \mathrm{L}^{3}$ Women with iron deficiency having low hemoglobin were termed as Iron deficiency anemia. In the newborns, iron deficiency was described as serum ferritin less than $75 \mu \mathrm{g} / \mathrm{L}$. Ferritin level above $370 \mu \mathrm{g} / \mathrm{L}$ was suggestive of perinatal infection or inflammation. Hence these sample values were excluded from the study. ${ }^{9}$

\subsection{Data analysis}

The collected data was coded and entered on the IBM Statistical Package for Social Sciences (SPSS) for Windows, version 25.0. Armonk, NY: IBM Corp. Neonatal parameters, including gender and SGA status, were summarized as mean and percentage. Factors associated with SGA was described as relative risk with $95 \%$ confidence interval. A p value of less than 0.05 was statistically significant. The neonatal anthropometry, ferritin, and maternal age, anthropometry, and hemoglobin were calculated as mean and standard deviation. Serum ferritin was characterized by median and interquartile range. The maternal factors like occupation, anemia status and the correlation between maternal anthropometry, maternal anemic status markers, neonatal anthropometry, and anemic markers were analyzed using the Pearson coefficient.

\section{Results}

\subsection{General characteristics}

Between May 2018 and August 2018, there were 1243 deliveries and 88 babies were included in the study. The data on the mother's anthropometry and weight gain were collected retrospectively from the records.

Neonatal features: The newborns born were divided into two groups, SGA as cases $(n=37)$ and AGA group as control $(n=51)$ (Table 1). Fifty-eight percent of the babies were AGA. Low birth weight was more in female babies as their male counterparts. The mean birth weight overall was adequate.

Maternal features: The mothers were mid-twenties, with fewer teenage pregnancies (Table 1). The patients had 11 years of mean schooling, with 67 of them have completed high school. All primigravida had four antenatal visits. Ninety-one percent of the women had been compliant to IFA supplementation. All women were, however, aware and had been provided with supplementation. The majority ( $\mathrm{n}=63$ ) were homemakers by profession. The mean weight gain 
Table 1

Description of neonatal and maternal characteristics.

\begin{tabular}{ll}
\hline Parameters & Mean (SD) \\
Neonatal Characteristics: & $2.69(0.5)$ \\
Birth Weight $(\mathrm{Kg})$ & $46.92(2.5)$ \\
Length $(\mathrm{cm})$ & $32.88(1.4)$ \\
Head Circumference (cm) & $51(58 \%)$ \\
Appropriate for Gestational Age babies(\%) & $37(42 \%)$ \\
Small for Gestational Age babies(\%) & \\
Maternal Characteristics: & $25.75(4.1)$ \\
Age(years) & 38 weeks one days(6) \\
Period of Gestation (weeks) & $80(91 \%)$ \\
Iron and folic acid supplementation $>$ 90days & $11(3.1)$ \\
Education (school years) & \\
Occupation n (\%) & $63(73.3)$ \\
Housewife & $15(17.4)$ \\
Unskilled work & $8(9.3)$ \\
Office Work & \\
Maternal anthropometry & $41.76(3.5)$ \\
Pre-pregnancy weight (Kg) & $10.01(4.23)$ \\
Weight gain during current pregnancy (Kg) & $152.68(6.9)$ \\
Height(cm) & $18(2.1)$ \\
Pre-pregnancy Body Mass Index(kg/m2) & \\
\hline
\end{tabular}

during pregnancy was $10 \mathrm{~kg}$ with more than a quarter of them $(\mathrm{n}=25)$ having a mean weight gain of less than $7 \mathrm{~kg}$. Only a third of them ( $\mathrm{n}=31$ ) had a weight gain $>12.5 \mathrm{~kg}$ as per Institute of Medicine pregnancy recommendations. ${ }^{10}$

\subsection{Factors affecting fetal growth}

\section{i) Maternal anemia:}

Sixteen SGA babies(43\%) had mothers with anemia.Thirteen babies had mothers with low serum ferritin,8(61.5\%) of them being AGA. Three out of 9 babies born with depleted iron stores were SGA. The BMI and weight gain during pregnancy were comparable in mothers with and without anemia.

The results were not statistically significant. However, it was observed an astonishingly high number of women were anemic (Table 2). Twenty-one (70\%) mothers had moderate anemia, with seven $(23 \%)$ having mild anemia. The serum ferritin, however, for most of the women was within the normal range. The serum ferritin of the babies also showed a similar picture (Table 3 ).

ii) Maternal Periconceptional BMI:

Periconceptional BMI did not play an essential role in birth weight (Table 3).

\section{iii) Maternal Weight Gain}

Poor maternal weight gain was characteristically related associated with low birth weight and smaller head circumference. The weight gain $>7 \mathrm{~kg}$ was observed to be associated with better fetal growth (Table 4).

\section{Discussion}

The mothers of the babies included in the study had malnutrition, defined based on their pre-pregnancy weight. Maternal malnutrition has been a long debatable cause of intrauterine growth restriction in babies. The National Family Health Survey IV (NFHS IV) of 2015-2016 states that $22.9 \%$ of women in the childbearing age group have BMI which is below normal. ${ }^{4}$ The burden of undernutrition in Dakshina Kannada, the district where the study was conducted, is a staggering $25.6 \%$ despite the female literacy rate of $80.3 \%$ against a national average of $68.3 \% .{ }^{11}$ However, NFHS IV has defined undernutrition in
Table 2

Comparision of maternal characters among SGA and AGA babies.

\begin{tabular}{|c|c|c|c|c|}
\hline \multirow[t]{2}{*}{ Parameter } & \multicolumn{2}{|c|}{ Babies included } & \multirow[t]{2}{*}{ RR (95\% CI) } & \multirow[t]{2}{*}{$P$ value } \\
\hline & SGA $n(\%)$ & AGA $n(\%)$ & & \\
\hline \multicolumn{5}{|c|}{ Maternal Characteristics: } \\
\hline \multicolumn{5}{|l|}{ Age group(yrs) } \\
\hline $18-25$ & $22(47.8)$ & $24(52.2)$ & 1.00 & \\
\hline 26-30 & $6(20)$ & $24(80)$ & $0.42(0.19-0.91)$ & 0.01 \\
\hline$>31$ & $9(75)$ & $3(25)$ & $1.57(1.01-2.45)$ & 0.11 \\
\hline \multicolumn{5}{|c|}{ Gestational age(weeks) } \\
\hline $37-38$ & $26(47.3)$ & $29(52.7)$ & 1.00 & \\
\hline $38.1-39$ & $6(26.1)$ & $17(73.9)$ & $0.55(0.26-1.16)$ & 0.08 \\
\hline$>39.1$ & $5(50)$ & $5(50)$ & $1.06(0.54-2.09)$ & 0.87 \\
\hline \multicolumn{5}{|c|}{ Pre pregnancy BMI $\left(\mathrm{kg} / \mathrm{m}^{2}\right)$} \\
\hline$<18.5$ & $19(38)$ & $31(62)$ & $0.80(0.49-1.31)$ & 0.38 \\
\hline$>=18.5$ & $18(47.4)$ & $20(52.6)$ & 1.00 & \\
\hline \multicolumn{5}{|l|}{ Weight gain (kg) } \\
\hline Mean & $8.54(3.95)$ & $11.07(4.15)$ & & 0.005 \\
\hline$<7$ & $16(64)$ & $9(36)$ & $1.92(1.22-3.03)$ & 0.0086 \\
\hline$>=7$ & $21(33.3)$ & $42(66.7)$ & 1.00 & \\
\hline \multicolumn{5}{|c|}{ Maternal Anemia(g/dL) } \\
\hline Anemic mothers & $16(53.3)$ & $14(46.7)$ & $1.47(0.91-2.38)$ & 0.12 \\
\hline Non-anemic mothers & $21(36.2)$ & $37(63.8)$ & 1.00 & \\
\hline \multicolumn{5}{|c|}{ Maternal Ferritin $(\mu \mathrm{g} / \mathrm{L})$} \\
\hline$<30$ & $5(38.5)$ & $8(61.5)$ & $1.51(0.68-3.40)$ & 0.33 \\
\hline$>=30$ & $16(25.4)$ & $47(74.6)$ & 1.00 & \\
\hline \multicolumn{5}{|l|}{ Sex of the Baby } \\
\hline Female & $21(53.8)$ & $18(46.2)$ & $1.65(1.00-2.71)$ & 0.045 \\
\hline Male & $16(32.7)$ & $33(67.3)$ & 1.00 & \\
\hline \multicolumn{5}{|l|}{$\operatorname{Cord~Ferritin~}^{\mathrm{a}}(\mu \mathrm{g} / \mathrm{L})$} \\
\hline$<75$ & $3(33.3)$ & $6(66.7)$ & $0.77(0.29-2.05)$ & \\
\hline$>=75$ & $22(43.1)$ & $29(56.9)$ & 1.00 & 0.72 \\
\hline
\end{tabular}

Significant $\mathrm{P}$ value $>0.05$.

BMI: Body mass index, AGA: Appropriate for gestational age, SGA: Small for gestational age.

a 12 mothers and 28 babies had serum Ferritin in inflammatory range and were excluded from the analysis.

Table 3

An overview of maternal anemia and ferritin and effect on the neonate.

\begin{tabular}{lll}
\hline Parameters & Anemic Mothers $n=30$ & $\begin{array}{l}\text { Non Aaemic Mothers } \\
n=58\end{array}$ \\
\hline Hemoglobin $(\mathrm{g} / \mathrm{dL})$ & $9.09(1.17)$ & $12.4(0.8)$ \\
${\text { Maternal Ferritin }(\mu \mathrm{g} / \mathrm{L})^{\mathrm{a}}}$ & $52(25.5-100.5)$ & $117(74-177)$ \\
${\text { Cord Ferritin }(\mu \mathrm{g} / \mathrm{L})^{\mathrm{a}}}_{\text {Weight gain }(\mathrm{kg})}$ & $166(68.5-247.75)$ & $201(120-288)$ \\
Pre-Pregnancy BMI$(\mathrm{kg} /$ & $9.5(4.3)$ & $10.3(4.2)$ \\
$\left.\quad \mathrm{m}^{2}\right)$ & $17.6(1.9)$ & $18.3(2.2)$ \\
Birth Weight $(\mathrm{kg})$ & $2.62(0.41)$ & $2.73(0.48)$
\end{tabular}

a Values of ferritin have been expressed as median with interquartile range. Other parameters have been explained as mean with standard deviation.

terms of BMI of less than $18.5 \mathrm{~kg} / \mathrm{m}^{2}$.Since the BMI changes during pregnancy, and there is no cut off values for BMI increase during pregnancy, a pre-pregnancy weight along with weight gain were preferred for defining the maternal undernutrition in the study. The largest drawback of having pre-pregnancy BMI as a marker for the SGA babies is the ample evidence stating that Asians are smaller in size and have less weight gain during pregnancy as their Caucasian counterparts. The BMI cut off levels are based on western literature. Our study did not find an association between lower pre-pregnancy BMI and low birth weight $(\mathrm{RR}=0.80, \mathrm{p}=0.38)$. A larger study in China that included 512 mother-neonate pairs had similar results. $(\mathrm{OR}=0.94, \mathrm{p}=0.40) .{ }^{12}$

The studies done so far from the Asian continent has been contradicting. The studies from Japan show no association between undernutrition and low birth weight. ${ }^{13}$ However, a Chinese study with a larger sample $(292,568)$, respectively, showed maternal undernutrition and inadequate weight gain were the risk factors for low birthweight 
Table 4

Influencing variables associated with neonatal anthropometry and ferritin.

\begin{tabular}{|c|c|c|}
\hline Comparison parameters & Correlation (r) & $P$ value \\
\hline \multicolumn{3}{|l|}{ Maternal parameters Vs. Anemia markers in mother } \\
\hline Pre pregnancy Weight Vs. Hemoglobin & 0.21 & 0.04 \\
\hline Pre pregnancy Weight Vs. Ferritin & 0.06 & 0.6 \\
\hline Pre pregnancy BMI Vs. Hemoglobin & 0.17 & 0.11 \\
\hline Pre pregnancy BMI Vs. Ferritin & -0.04 & 0.8 \\
\hline Weight gain Vs. Ferritin & -0.042 & 0.72 \\
\hline \multicolumn{3}{|l|}{ Maternal Vs. fetal anemia markers } \\
\hline Maternal Hemoglobin Vs. Cord Ferritin & 0.22 & 0.09 \\
\hline Maternal Ferritin Vs. Cord Ferritin & -0.08 & 0.44 \\
\hline \multicolumn{3}{|l|}{ Maternal anemia markers with birth weight } \\
\hline Hemoglobin Vs. birth weight & 0.13 & 0.22 \\
\hline Ferritin Vs. birth weight & 0.23 & 0.04 \\
\hline \multicolumn{3}{|l|}{ Maternal anemia marker with neonatal length } \\
\hline Hemoglobin Vs. length & -0.02 & 0.84 \\
\hline Ferritin Vs. length & -0.07 & 0.53 \\
\hline \multicolumn{3}{|c|}{ Maternal anemia marker with neonatal head circumference } \\
\hline Hemoglobin Vs. head circumference & 0.12 & 0.25 \\
\hline Ferritin Vs. head circumference & -0.11 & 0.34 \\
\hline \multicolumn{3}{|l|}{ Cord blood ferritin with neonatal anthropometry } \\
\hline Ferritin Vs. birth weight & -0.13 & 0.31 \\
\hline Ferritin Vs. length & 0.14 & 0.3 \\
\hline Ferritin Vs. head circumference & -0.09 & 0.48 \\
\hline \multicolumn{3}{|l|}{ Maternal weight gain Vs. neonatal characteristics } \\
\hline Maternal weight gain Vs. birth weight & 0.41 & 0.0001 \\
\hline Maternal weight gain Vs. length & 0.18 & 0.08 \\
\hline Maternal weight gain Vs. head circumference & 0.33 & 0.0015 \\
\hline Maternal weight gain Vs. cord ferritin & 0.008 & 0.95 \\
\hline
\end{tabular}

Significant Correlation $>0.30$, Significant $\mathrm{P}$ value $>0.05$.

and SGA infants. ${ }^{14}$

The cause of SGA babies as per the study is reduced weight gain ( $\mathrm{p}<0.001)$.Similar studies from Vietnam $(\mathrm{n}=2692, \mathrm{OR}=1.9,95 \% \mathrm{CI}$ 1.48-2.44,p $<0.001)$ and China(n $=512$, OR $=3.6, \mathrm{p}<0.01)$ support lower maternal weight gain as a risk factor for low birth weight. $^{15,16}$

A Swedish study has used a sibling comparison approach for deducing poor weight gain as a causal agent for low birth weight. The results were adjusted using a hybrid fixed effect model that nullified the effect of genetic and time-independent environmental factors. The between-pregnancy analysis observed to have a gross difference of risk ratio by 0.70 for every one z-score difference in pregnancy weight gain, irrespective of the BMI. The study observed interestingly that the pattern of weight gain did not make an effect on fetal growth. The study provides substantial evidence to the findings of our study and validates the effect of adequate pregnancy weight gain for a larger population. ${ }^{17}$

The proportion of mothers having anemia in the study was $34 \%$, which is significantly high compared to the fact that the mothers were on adequate supplementation and deworming had been done once. The malaria status was negative. WHO recommends 30-60 mg iron and dose to be increased to $120 \mathrm{mg}$ if anemia is diagnosed and to be continued till improvement. Macrocytic anemia associated with low folate levels should be treated with $1 \mathrm{mg}$ of oral folate tablets. This could be one of the reasons in the failure to eliminate anemia in pregnancy as a major health problem, as none of the mothers had been screened for the cause of the anemia. ${ }^{18,19}$

The national data puts the onus on poor compliance(30.3\%)with oral IFA tablets, unlike our study, where $91 \%$ of mothers regularly consumed the tablets. ${ }^{4}$ This disproportionate gap between micronutrient supplementation and maternal anemia can be explained by social factors including poor sanitation, lack of awareness of the cooking practices, nutritional values of the food being consumed and protocol driven approach of all nutritional programs than understanding the needs of each mother. The cause of anemia needs to be evaluated further and treated by behavior change communication along with supplementation. A study from Varanasi supports that by effective communication with the mother, family participation, home-based reminder, and remediation the hemoglobin status could improve and the proportion of anemia was reduced by half. ${ }^{20}$ Another multi-center based randomized controlled trial, which included a lipid-based micronutrient supplement given to mothers during the periconceptional period as intervention, reported the benefit to the fetus in terms of better linear growth and weight. A major similarity to our study was the baseline biochemical evidence was not taken into consideration during enrollment of the participants. The study noted that intervention in the periconceptional period was not statistically superior to intervention in the late first trimester. Hence, adequate weight gain if achieved in pregnancy can break the cycle of alternating maternal and fetal malnutrition. $^{21}$

Anemia in pregnancy has been long since associated with low birth weight. The results of our study, like other studies, point toward an ambiguous picture. ${ }^{22,23}$ There was no statistic correlation. However, the proportion of anemic mothers with SGA babies was higher.

The most common type of anemia prevalent among pregnant mothers is secondary to iron deficiency. During pregnancy, there is an increased need for iron for fetal growth. Iron plays a prominent role in growth, cognition, and psychomotor development of the fetus and infancy period. The prospect of using serum ferritin as a single screening marker for iron deficiency was chosen because the iron stores get depleted before the anemia sets. ${ }^{24}$ Our study found an uncommonly fewer number of women to have iron deficiency anemia, whereas only one mother had lower ferritin level without lower hemoglobin levels. The reason was that almost all women had been supplemented with IFA tablets.

The maternal ferritin showed a significant correlation with birth weight, accentuating the importance of iron stores on fetal growth. A recent cross-sectional study on Palestinian women links iron depletion in the first trimester with low birth weight. The maternal anthropometry did not influence neonatal growth, but the study did not include mean weight gain as an influential factor. ${ }^{25}$ There was no correlation between maternal and neonatal cord ferritin in our study. The consequence of anemia in pregnancy on growing fetus could not be precisely concluded as hemoglobin was measured on admission for delivery, rather than serial values throughout gestation.

\section{Conclusion}

The desirable fetal growth in malnourished mothers can be achieved despite maternal anemia with adequate weight gain. The pre-pregnancy BMI does not play a significant role in fetal growth but may have farreaching outcomes. The present IFA supplementation program have not yet been effective in reducing maternal anemia. The problem of low birth weight is complex and needs to be analyzed to achieve a favorable outcome in infant mortality reduction.

\section{Funding}

None.

\section{Declaration of competing interest}

There is no potential conflict of interest.

\section{Acknowledgments}

We want to thank Dr. Savitha, Medical Superintendent and Dr. Murali Keshava, Assistant Professor and In-charge of Lady Goschen Hospital, Mangaluru for permitting for the study to be carried out in premises of Lady Goschen Hospital. We thank Dr. Kamalakshi G. Bhat, Head of Department, Pediatrics, Kasturba Medical College, Mangalore, for her guidance and extend our gratitude to Miss Disha, medical social worker, for her help with data collection. 


\section{References}

1. Kramer MS. Determinants of low birth weight: methodological assesment and metaanalysis. Bull WHO. 1987;65(5):663-737.

2. Barker DJP, Godfrey KM, Gluckman PD, Harding JE, Owens JA, Robinson JS. Fetal nutrition and cardiovascular disease in adult life. Lancet. 1993;341:938-941.

3. National Iron + Initiative Guidelines for Control of Iron Deficiency Anaemia. 2013; 2013.

4. International Institute for Population Sciences. National Family Health Survey-4, State Fact Sheet, 2015 -16: India. Mumbai: IIPS; 2016.

5. Ververs MT, Antierens A, Sackl A, Staderini N, Captier V. Which anthropometric indicators identify a pregnant woman as acutely malnourished and predict adverse birth outcomes in the humanitarian context? PLoS Curr. 2013;5.

6. World Health Organisation. Obesity: Preventing and Managing the Global Epidemic WHO Technical Report Series 894 Geneva: World Health Organization; 2000.

7. Coffey D. Prepregnancy body mass and weight gain during pregnancy in India and sub-Saharan Africa. Proc Natl Acad Sci U S A. 2015;112(11):3302-3307.

8. American College of Obstetricians and Gynaecologists. Anemia in pregnancy.ACOG practice bulletin No.95. Obstet Gynecol. 2008;112(1):201-207.

9. Shao J, Lou J, Rao R, et al. Maternal serum ferritin concentration is positively associated with newborn iron stores in women with low ferritin status in late pregnancy. J Nutr. 2012;142(1):2004-2009.

10. Institute of Medicine, Nutrition Research Council. Rasmussen KM, Yaktine AL, eds. Weight Gain during Pregnancy: Reexamining the Guidelines [Internet]. Washington, DC: The National Academies Press; 2009:254-255.

11. International Institute for Population Sciences. District Level Household \& Facility Survey (DLHS-4). Mumbai: IIPS; 2016.

12. Xiao L, Ding G, Vinturache A, et al. Associations of maternal pre-pregnancy body mass index and gestational weight gain with birth outcomes in Shanghai, China. Sci Rep. 2017;7.

13. Akahoshi E, Arima K, Miura K, et al. Association of maternal pre-pregnancy weight, weight gain during pregnancy, and smoking with small-for-gestational-age infants in Japan. Early Hum Dev. 2016;92:33-36.
14. Liu Y, Dai W, Dai X, Li Z. Prepregnancy body mass index and gestational weight gain with the outcome of pregnancy: a 13-year study of 292,568 cases in China. Arch Gynecol Obstet. 2012;286:905-911.

15. Ota E, Haruna M, Suzuki M, et al. Maternal body mass index and gestational weight gain and their association with perinatal outcomes in Viet Nam. Bull World Health Organ. 2011;89:127-136.

16. McDonaldChristy SD, Woolcott C, Chapinal N, Yanfang G, Murphy P, Dzakpasu S. Interprovincial variation in pre-pregnancy body mass index and gestational weight gain and their impact on neonatal birth weight with respect to small and large for gestational age. Can J Public Health. 2018;109(4):527-538.

17. Hutcheon JA, Stephansson O, Johansson K, Cnattingius S, Bodnar LM. Is the association between pregnancy weight gain and fetal size causal? Epidemiology. 2018;30(2):234-242

18. World Health Organisation. Daily Iron and Folic Acid Supplementation in Pregnant Women. Geneva: WHO Press; 2012:4-5.

19. Annamraju H, Pavord S. Anemia in pregnancy. Br J Hosp Med. 2016;77(10):584-588.

20. Shivalli S, Srivastava RK, Singh GP. Trials of improved practices (TIPs) to enhance the dietary and iron-folate intake during pregnancy- A quasi experimental study among rural pregnant women of Varanasi, India. PLoS One. 2015;10(9):e0137735.

21. Hambidge KM, Westcott JE, Garcés A, et al. A multicountry randomized controlled trial of comprehensive maternal nutrition supplementation initiated before conception : the Women First trial. Am J Clin Nutr. 2019;109:1-12.

22. Srinivas $P$, Srinivasan $P$. The relationship between maternal anemia and birth weight in new born. IOSR J Dent Med Sci. 2015;14(12):9-11 e-ISSN.

23. Sá SA De, Willner E, Aguiar T, et al. Anemia in pregnancy : impact on weight and in the development of anemia in newborn. Nutr Hosp. 2015;32(5):2071-2079.

24. Mireku MO, Davidson LL, Boivin MJ, et al. Prenatal iron deficiency, neonatal ferritin, and infant cognitive function. Pediatrics. 2016;138(6):e20161319.

25. Srour MA, Aqel SS, Srour KM, Younis KR, Samarah F. Prevalence of anemia and iron deficiency among Palestinian pregnant women and its association with pregnancy outcome. Anemia. 2018:9135625. 